\title{
Polimorfismo de fármacos no controle de qualidade de medicamentos: uma revisão bibliográfica
}

\author{
Drug polymorphism in the drug quality control: a review
}
Polimorfismo de fármacos en el control de calidad de medicamentos: una revisión bibliográfica

\begin{abstract}
Alexsander Augusto da Silveira ${ }^{1 *}$, Amanda Evely Teixeira Pereira ${ }^{1}$, Ikaro Silva de Oliveira ${ }^{1}$, Flávio Silva de Carvalho², Adibe Georges Khouri, Álvaro Paulo Silva Souza ${ }^{1}$.
\end{abstract}

\section{RESUMO}

Objetivo: Revisar na literatura sobre os testes de identificação do polimorfismo de fármacos, a importância desse fenômeno no desenvolvimento de fármacos para a indústria farmacêutica e demonstrar os estudos mais recentes e atuais de fármacos que possuem estruturas polimórficas. Método: $O$ artigo foi realizado a partir da análise de artigos científicos, com a seleção de artigos diretamente ligado ao tema. Resultado: 0 polimorfismo, dependendo das aplicações e alterações físico -químicas impacta diretamente na produção de fármacos no estado sólido, com alterações na biodisponibilidade, pode apresentar efeito placebo e efeito tóxico. Dependendo do fármaco, das condições de produção, como pH e temperatura e do excipiente utilizado no desenvolvimento da forma farmacêutica, diferentes formas de polimorfos do mesmo fármaco podem ser observadas. Considerações Finais: O Brasil tem a sua produção farmacêutica baseada na produção de medicamentos genéricos e similares, desta forma, formulações, excipientes e o processamento são adaptados para uma nova demanda. Dependendo das condições de obtenção e produção novas estruturas no estado sólido podem ser observadas, o que exige um melhor monitoramento pelo controle de qualidade de medicamentos nas indústrias farmacêuticas. Novos princípios ativos e otimizações de fármacos podem ser explorados por este processo. Trabalhos nessa área são escassos, novos estudos e descrições são imprescindíveis.

Palavras-chave: Polimorfismo, Drogas, Cristalização, Biodisponibilidade.

\begin{abstract}
Objective: To review the literature about drug polymorphism, the importance of this phenomenon in the development of drugs for the pharmaceutical industry and to demonstrate the most recent and current studies of drugs that have polymorphic structures. Method: The paper was carried out from the analysis of scientific articles, selecting those with content directly related to the theme. Result: Polymorphism, depending on the applications and physical-chemical properties directly impacting the production of drugs in the solid state, with changes in bioavailability, may present a placebo effect and toxic effect. Depending on the drug, the production conditions, such as $\mathrm{pH}$ and temperature and the excipient used in the development of the pharmaceutical form, different structures of polymorphs of the same drug can be observed. Final Considerations: Brazil has its pharmaceutical production based on the production of generic drugs and the like, in this way, formulations, excipients and processing are adapted to a new demand. Depending on the conditions of production and production new properties in the solid state can be observed, which requires better monitoring by the quality
\end{abstract}

${ }^{1}$ Faculdade Estácio de Sá de Goiás, Goiânia-GO. *E-mail: alekfarm2000@yahoo.com.br

2 Universidade Federal de Goiás, Instituto de Química, Faculdade de Farmácia, Goiânia-GO.

SUBMETIDO EM: 4/2019

ACEITO EM: 5/2019

PUBLICADO EM: $8 / 2019$

REAS/EJCH | Vol. Sup. 29 | e791 | DOI: https://doi.org/10.25248/reas.e791.2019 Página 1 de 8 
control of medicines. New active principles and drug optimization can be exploited by this mechanism. Studies in this area are scarce and descriptions are imperative.

Key words: Polymorphism, Drugs, Crystallization, Bioavailability.

\section{RESUMEN}

Objetivo: Revisar en la literatura sobre las pruebas de identificación del polimorfismo de fármacos, la importancia de este fenómeno en el desarrollo de fármacos para la industria farmacéutica y demostrar los estudios más recientes y actuales de fármacos que poseen estructuras polimórficas. Método: Fue realizado a partir del análisis de artículos científicos, seleccionando aquellos con contenido directamente ligado al tema. Resultado: El polimorfismo, dependiendo de las aplicaciones y alteraciones físico -químicas impacta directamente en la producción de fármacos en estado sólido, con alteraciones en la biodisponibilidad, puede presentar efecto placebo y efecto tóxico. Dependiendo del fármaco, de las condiciones de producción, como $\mathrm{pH}$ y temperatura y del excipiente utilizado en el desarrollo de la forma farmacéutica, diferentes formas de polimorfos del mismo fármaco pueden ser observadas. Consideraciones finales: Brasil tiene su producción farmacéutica basada en la producción de medicamentos genéricos y similares, de esta forma, formulaciones, excipientes y el procesamiento son adaptados para esa demanda. Dependiendo de las condiciones de obtención y producción nuevas estructuras en el estado sólido pueden ser observadas, exigiendo un mejor monitoreo por el control de calidad de medicamentos. Los nuevos principios activos y las optimizaciones de fármacos pueden ser explotados por este proceso. Los trabajos en esta área son escasos, nuevos estudios y descripciones son imprescindibles.

Palabras clave: Polimorfismo, Drogas, Cristalización, Biodisponibilidad.

\section{INTRODUÇÃO}

Em 1821, o químico alemão Eilhard Mitschelic observou pela primeira vez o polimorfismo. Já em 1940, Mc Crone, definiu polimorfismo como compostos orgânicos que possuem uma ou mais formas cristalinas podendo ser encontrados em sete diferentes tipos de sistemas cristalinos fundamentais i.e. monoclínico, triclínico, ortorrômbico, tetragonal, trigonal, hexagonal ou cúbico. Apesar de ter sido descoberto há mais de dois séculos, atualmente é um assunto frequentemente abordado pelo setor farmacêutico (ARAUJO GLB, et al., 2012).

O polimorfismo termo do grego poly (muitos), morphos (formas), a partir de uma arquitetura de interações intermoleculares e intramoleculares no estado sólido, os fármacos apresentam diferentes propriedades físicoquímicas tais como, solubilidade, ponto de fusão, densidade, dureza, comportamento térmico e pressão de vapor. Estas diferentes propriedades estão relacionadas com as divergências das formas cristalinas em uma mesma composição química (CASTRO RAE, et al., 2013).

Em alguns sistemas de cristalização existem diferentes formas sólidas, a cristalina, que é uma forma identificável e de estrutura bem definida, ou a forma amorfa, a qual é uma forma que implica em uma desordem a nível molecular. A forma cristalina é dividida em polimorfos e pseudopolimorfos, onde o polimorfo possui uma entidade ímpar que é estável a uma determinada temperatura e pressão. A estabilidade, apresentada pelos polimorfos, faz com que o fármaco apresente um ponto de fusão mais elevado e uma solubilidade mais baixa, ou seja, garantindo toda arquitetura físico-química do fármaco durante seu processo de armazenamento e consumo (DUTRA KCS, 2005; BORTOLLUZZI ALML, 2011).

A detecção de polimorfismo para compostos orgânicos com a mesma formulação química e com estruturas cristalinas diferentes no estado sólido dá se por meio de métodos instrumentais analíticos como espectroscopia de infravermelho (IR); ressonância magnética nuclear (RMN); difração de raios $X$ por poli cristais (DRX); calorimetria exploratória diferencial (DSC), termogravimetria (TG), etc. A formação de polimorfos pode ocorrer na matéria prima (princípio ativo), na produção e/ou no armazenamento do fármaco 
(ARAUJO, GLB et al., 2012). A Figura 1 apresenta um fluxograma das etapas de formação e as principais metodologias instrumentais para identificação do processo polimórfico.

Figura 1 - Possíveis apresentações da estrutura cristalina de um composto químico.

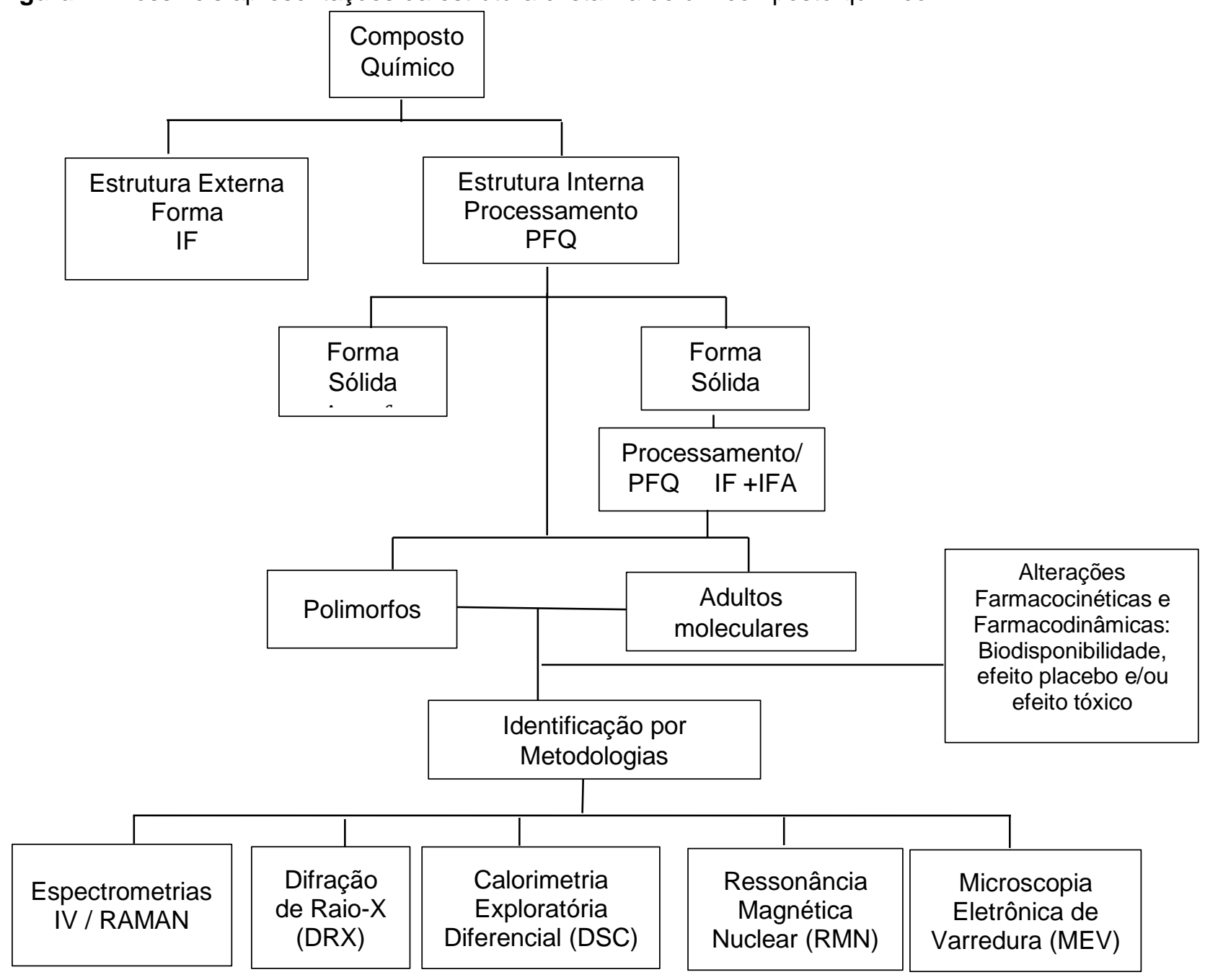

Fonte: Próprios autores, 2019.

Legenda: IF- Insumo Farmacêutico; IFA- Insumo Farmacêutico Ativo; PFQ- Propriedades físico-químicas;

É uma necessidade da indústria farmacêutica, obter o controle das propriedades físico-químicas e farmacocinéticas dos fármacos polimorfos, pois, isto implica diretamente na garantia de qualidade dos fármacos nas formas sólidas, assim como na sua farmacodinâmica ao seu consumidor final. O polimorfismo impacta diretamente na produção de fármacos no estado sólido, uma vez que altera a biodisponibilidade comprometendo assim a velocidade de dissolução e a absorção (LOZANO LS, 2005). Desta forma, o objetivo dessa revisão de literatura é descrever o mecanismo de polimorfismo de fármacos, com apresentação dos testes de identificação deste processo físico-químico dos fármacos, assim como demonstrar os estudos mais recentes e atuais de fármacos que possuem estruturas polimórficas.

\section{MÉTODOS}

Trata-se de uma revisão de literatura exploratória, realizada por meio de consultas nas bases de dados da PubMed, Scientific Electronic Library Online (SciELO), Centro Latino-Americano e do Caribe de Informação em Ciências da Saúde- BIREME e Biblioteca Virtual da Saúde (BVS). Para a seleção dos artigos foram 
utilizados como descritores para a pesquisa os termos polimorfismo, fármacos, identificação química e biodisponibilidade. Foram incluídos e selecionados para o desenvolvimento do estudo de revisão, principalmente estudos dos anos de 2005 à 2016, além dos artigos clássicos relacionados ao assunto proposto nos idiomas português e inglês.

\section{RESULTADOS E DISCUSSÃO}

\section{Fármacos que apresentam formas polimorfas}

O primeiro caso de polimorfismo em fármacos foi descrito com o ritonavir, um medicamento antirretroviral inibidor da enzima protease viral, utilizado no tratamento de pacientes HIV-1 positivos. Esse medicamento foi disponibilizado para o mercado na data descrita, em duas formas farmacêuticas, o ritonavir líquido e o ritonavir em cápsulas gelatinosas sem o conhecimento do processo de polimorfismo. Durante o processo de desenvolvimento do ritonavir cápsulas, somente uma forma cristalina foi identificada e todas elas sem problemas de estabilidade. No entanto, em 1998, grande parte dos fármacos ritonavir cápsulas apresentaram falhas em sua dissolução, com diminuição da biodisponibilidade do fármaco, com consequente alteração da farmacodinâmica. Devido a esse fato os cristais deste lote foram analisados pelo método de DRX, foi observado a presença de duas formas cristalinas, I e II, em que a forma II possuía alguns picos no difratograma ausentes na forma I (BAUER J, et al., 2001).

A forma II identificada, posteriormente melhor estudada e elucidada apresenta uma baixa solubilidade em solventes convencionais da formulação, que pela técnica de MEV observa se a presença de ambos os cristais, com aspecto cristalino totalmente diferente da forma I. Essas transformações ocorrem por causa de falhas no procedimento de cristalização e de purificação, no qual após refazerem as análises foram identificadas impurezas nos solventes e resíduos de carbamato de etila. Desde então, métodos de preparação da forma farmacêutica utiliza pequenas quantidades de sementes e técnica inversa da adição. Trabalhando em menor escala de solução e com adição de sementes obteve-se apenas o polimorfo I meta-estável, além disso observa - se que a adição de um solvente apropriado elimina a formação do polimorfo II (BAUER J, et al., 2001).

Outro exemplo de fármaco com características polimórficas é o mebendazol, utilizado para tratamento de infecções simples ou mistas por helmintos das espécies Enterobius vermicularis, Trichuristrichuria, Ascaris lumbricóides, Ancylostomaduodenale e Necator americanus. O ativo mebendazol apresenta três formas cristalinas diferentes $A, B$ e $C$, sendo que nestas três formas os comportamentos físicos, físico-químicos e tóxicos são diferentes. Estudos subsequentes realizados confirmaram que a forma $C$ é a mais eficaz para 0 tratamento anti-helmíntico; a forma cristalina B apresenta maior toxicidade e a forma cristalina A é a menos tóxica, porém é menos solúvel e menos lipofílica o que acarreta numa grande dificuldade na absorção e biodisponibilidade desta forma cristalina (CAABEIRO RF, et al., 1987; COSTA J, et al., 1991; FROEHLICH PE e GASPAROTTO FS, 2005; BORTOLLUZZI ALML, 2011).

Outro tipo de polimorfismo a ser citado é o apresentado pela tibolona, um fármaco para tratamento de distúrbios da menopausa natural ou cirúrgica, no qual podem apresentar suas formas I (monoclínica) e a forma II (triclínica). A forma I possui alongamento de cristais em solventes polares como acetonitrila e acetona, já a forma II alongamento de cristais em solventes apolares, como $n$ - hexeno e tolueno. As formações desses cristais se diferenciam na supersaturação, mas pode acontecer de ambas as formas apresentarem cristalização no mesmo solvente e temperatura, fenômeno que leva à cristalização concomitante das duas formas. Estudos de estabilidade da tibolona realizados por RMN, comprovam que não ocorre transição de fase durante o desenvolvimento dos fármacos na forma de comprimidos. Análises subseqüentes mostraram que a forma I deve ser utilizada na produção da tibolona, uma vez que o processo de recristalização pode levar a forma triclínica para a fase monoclínica. Além disso, a forma triclínica reduz a estabilidade físicoquímica do medicamento (BOERRIGTER SXM, 2002; ARAÚJO GLB, 2009).

A espironolactona é um fármaco de ações diuréticas, anti-hipertensivas que atua no túbulo renal distal onde eleva a eliminação de água, sódio e promove a retenção de potássio. No processo de recristalização da

REAS/EJCH | Vol. Sup. 29 | e791 | DOI: https://doi.org/10.25248/reas.e791.2019 Página 4 de 8 
espironolactona em diferentes solventes, como acetonitrila, etanol, clorofórmio e acetato de etila, apresenta duas formas polimórficas não solvatadas, I e II, detectadas pela técnica de cristalografia de raios-X. Os dois polimorfos, de estruturas ortorrômbicas, mas com diferenças em seus anéis esteróidais, quando submetidos a uma alta pressão apresentam diferenças consideráveis. A forma I, o polimorfo menos estável termodinamicamente, não se transforma no formato II, além disso sobre pressões ambas as estruturas estão susceptíveis a possíveis novos polimorfos. Na ausência de alta pressão, entretanto, é impossível apreciação da natureza das estruturas de transição destas moléculas. Estes resultados são importantes para indústria farmacêutica em termos de processamento da espironolactona, no qual pressões mecânicas podem ser aplicadas e imprescindível para identificar o polimorfismo presente neste processo de produção (PISEGNA GL; GILSON DFR e BUTLER IS, 2014). A Tabela 1 apresenta os fármacos com os seus principais polimorfos e as metodologias instrumentais de identificação.

Tabela 1 - Fármacos que apresentam polimorfismo e metodologias instrumentais de identificação.

\begin{tabular}{cccc}
\hline Fármacos & Polimorfos & $\begin{array}{c}\text { Forma Estável para } \\
\text { Indústria }\end{array}$ & $\begin{array}{c}\text { Metodologia/ } \\
\text { Identificação }\end{array}$ \\
\hline Carvedilol & $1,2,3$ e 4 & - & DRX, DSC, TG, MEV \\
Clortalidona & I, II e III & DRX \\
Fluconazol & I-IX & I & DSC, DRX, RAMAN \\
Mebendazol & A, B, C & C & DRX \\
Espironolactona & I e II & II & RAMAN \\
Ritonavir & I e II & I & DRX, MEV \\
Tibolona & I e II & I & RMN \\
Tenofovir & & & DRX, RAMAN
\end{tabular}

Fonte: Próprios autores, 2019.

O caverdilol utilizado para o tratamento de pacientes com hipertensão, insuficiência cardíaca leve a grave, infarto do miocárdio e angina do peito, devido a sua atividade farmacológica como antagonista $\beta$-adrenérgico não seletivo. O fármaco possui uma baixa solubilidade e uma alta permeabilidade, de modo que é praticamente insolúvel em água. Através das técnicas de identificação, o carvedilol foi caracterizado em diferentes solventes, por DRX, DSC, TG e MEV. Na análise dos cristais por DRX, novos picos foram elucidados quando comparado com o padrão, o que significa a formação de um novo cristal no estado sólido. $\mathrm{Na}$ análise por MEV observa se a diferenciação dos hábitos cristalinos, onde alguns cristais apresentam a forma de agulha e outros a forma de placas irregulares. A partir de processos de evaporação de solventes, foram encontrados quatro novos sais de caverdilol com ácidos orgânicos, como ácido oxálico, ácido fumárico, ácido benzoico e ácido mandélico. Os sais apresentados podem ser cristalizados de duas formas diferentes dependendo do solvente utilizado (HIENDRAWAN S, et al., 2016).

O fluconazol, antifúngico de amplo espectro de ação da classe dos imidazólicos tricíclicos e que apresenta várias vantagens em relação a outros antifúngicos, com um, menores efeitos colaterais e a possibilidade de atravessar a barreira hematoencefálica para tratamento de meningingites por Cryptoccocus. Este fármaco 
pode apresentar duas formas polimórficas que foram demonstradas pelas técnicas de DRX, espectroscopia de RAMAN e DSC. O fluconazol foi submetido a várias alterações de pressão, e foram identificados novos espectros de RAMAN, devido a um alongamento de vibrações do anel triazol. Após as análises de DRX e espectro RAMAN, foi observado que aplicações de diferentes pressões levam a uma transformação de fase polimórfica do fluconazol, que favorece a reorientação das moléculas devido à pressão (GORKOVENKO EA, et al., 2015).

O fumarato disoproxil de tenofovir (TDF) é um pró-fármaco utilizado no coquetel antirretroviral contra o HIV-1 e também para tratamento de hepatite B crônica, de forma que atua na inibição da enzima transcriptase reversa viral (TR). No desenvolvimento do TDF duas formas de polimorfismo são descritas, a formas I e a forma A, identificadas por técnicas de espectroscopia RAMAN e por DRX. Estudo recente demonstrou que no processo de preparo da formulação farmacêutica pode levar a conversão da forma I para a forma $A$, além disso a forma A possui pouca semelhança com a forma I. A forma $A$ é mais estável e possui menor solubilidade, já a forma I possui alta solubilidade e baixa permeabilidade, no entanto a transformação da forma I para a A ocorre involuntariamente durante o processo de fabricação, uma vez que o pH ácido influencia na transformação. Utilizando a granulação úmida de TDF ainda ocorria mudança para forma $A$, após a troca da granulação úmida por compreensão direta TDF identificaram que não promovia mais a transformação de forma I para a forma A (SLÁDKOVÁ V; DAMMER O e KRATOCHVÍL B, 2016).

\section{Desenvolvimento de fármacos e processos de cristalização}

O polimorfismo impacta diretamente no desenvolvimento de fármacos sólidos uma vez que altera a sua biodisponibilidade, assim como a velocidade de dissolução e a velocidade de absorção. Essas alterações nas propriedades físico-químicas, devido a diferentes estruturas cristalinas, podem promover alteração na eficácia do medicamento, não apresentar efeito algum (placebo), ou até mesmo se tornar tóxico ao paciente (GUTIERREZ SA, 2010). A síntese de medicamentos pelas Indústrias Farmacêuticas Químicas (IFQ) seguem em fluxo que se dá por meio de síntese, purificação, cristalização, secagem, cisalhamento e armazenamento de fármaco. Em decorrência dessa sequência é observado se há alguma alteração na composição, presença de solventes ou de substancias tóxicas, além de transformações que podem ocorrer durante um processo de cristalização. O método de cristalização ocorre por meio da volatilização de um líquido até a formação de cristais em solução saturada, sistema este que pode ser dividido de duas maneiras: por resfriamento, de forma que diminuindo a temperatura consegue obter uma substância menos solúvel, ou alterando a solução até que chegue a formar cristais, por controle da pressão e da temperatura (DUTRA KCS, 2005).

O método consiste em dissolver o soluto em um solvente até um ponto com acréscimo de mais soluto chegar a uma solução saturada. Esta metodologia pode desencadear o processo de nucleação no qual ocorre formação de cristais em solução supersaturada. A nucleação primária se dá por formação de núcleos através de choques entre moléculas em agitação numa solução saturada. Também pode ocorre nucleação secundária que é a formação de núcleos induzidos pelos cristais pré-existentes. Continuando com nucleação primária em que se ramifica em homogênea, que é a formação de núcleos espontaneamente, e heterogênea que é a formação de núcleos induzidos por superfície de contato com agentes externos, logo o crescimento é a junção de mais soluto ao núcleo formado (DUTRA KCS, 2005; MARTINS FT, 2010). Devido a importância do polimorfismo para a indústria farmacêutica durante todo o processo de desenvolvimento dos fármacos, foi determinado que a partir de 2007 os medicamentos genéricos e similares que possuírem algum polimorfismo deverá ser informado no momento do registro ou da renovação (pós-registro) desses fármacos perante a ANVISA, assim como registrar os métodos analíticos e os resultados encontrados de possíveis polimorfismos (CAPUCHO HC; MASTROIANNI PC e CUFFINI S, 2008).

\section{Técnicas para identificação de polimorfismo em fármacos}

Diversas técnicas são utilizadas para a identificação de polimorfismo em substâncias sólidas farmacêuticas. A técnica de espectrometria de IV permite identificar o tipo de grupo funcional presente na amostra, uma vez que se baseia em radiação eletromagnética na faixa do infravermelho, que ao passar pela amostra consegue mensurar as vibrações moleculares que ocorrem através das energias nas ligações

REAS/EJCH | Vol. Sup. 29 | e791 | DOI: https://doi.org/10.25248/reas.e791.2019 Página 6 de 8 
químicas. Análise essa que auxilia na identificação de um composto cristalizado se houver alteração em sua arquitetura química. Já a técnica de Raman identifica, também, se há variações na engenharia molecular de uma amostra a partir das vibrações das ligações, no entanto, diferentemente da espectroscopia de infravermelho que analisa as vibrações moleculares por absorção, analisa a frequência de incidência da radiação numa estrutura de conformação (DUTRA KCS, 2005; BORTOLLUZZI ALML, 2011).

A MEV serve para identificar o tipo de polimorfismo, uma vez que, pode-se definir o tamanho e a forma do cristal. A análise visual do cristal pode ser feita por microscópio óptico onde a visualização é mais limitada, uma vez que identifica apenas moléculas maiores, pois, a objetiva de maior grau é de 2.000 vezes. No entanto, a visualização pode ser feita também pelo microscópio eletrônico, que permite um grau de identificação eletrônico, muitas vezes maior do que o óptico (DUTRA KCS, 2005; ARAUJO GLB, 2009).

A DRX das formas cristalinas de fármacos tem como fundamento identificar, quantificar e determinar o estado físico dos cristais ou identificar o estado cristalino ou amorfo pelo desvio angular do cristal através dos raios-X emitidos sobre ele, uma vez que cada cristal após ser incendido por raios-X apresenta um ângulo de radiação especifico. A amostra deve ser preparada em forma de pó fino, pois, os raios- $X$ monocromático atingem a amostra dentro do detector na qual irá refletir raios- $X$ incidente por diversas direções, esses raios$X$ são gravados pelos métodos de Debey- Sherren ou de Guinier, ou pelo difratômetro. Esses raios incidentes são comparados com difrato gramas de substancia padrão, onde irá determinar a existência de polimorfismo através dos resultados obtidos a partir da inclinação do plano de cristal e da intensidade dos raios- $X$ que determina a distância entre o plano do cristal (LOZANO LS, 2005).

A análise térmica aplicada em estudos de pureza na caracterização de polimorfismo e também nas velocidades da degradação química de fármacos demonstra a interação da molécula do ativo com a substância que compõem a formulação. Existem duas técnicas que mais são aplicadas, a TG e a DSC. A técnica TG permite mensurar a massa através da variação de temperatura em um espaço controlado, já o DSC permite avaliar o fluxo de calor quando uma amostra for aquecida e resfriada (ARAÚJO GLB, 2009; MARTINS FT, 2010; MEIRELLES LMA, 2014).

Em um processo simultâneo entre TG/DSC pode se quantificar em uma mesma análise oscilações da massa e do calor de uma amostra. O TG divide-se em três tipos, TG isotérmica que é a variação da massa em função do tempo; TG quase-isotérmica onde a amostra será aquecida a uma temperatura constante até chegar a uma variação de massa e a TG dinâmica, na qual ocorre uma variação de temperatura préprogramada. O gráfico de TG possui curvas, que indicam variações das massas por evaporação de água, reações químicas ou a partir de estudos de reações químicas, volatilização, adsorção e dessorção. (ARAÚJO GLB, 2009; MARTINS FT, 2010; MEIRELLES LMA, 2014).

A RMN é uma técnica que se baseia na absorção de radiação em uma frequência de rádio do espectro eletromagnético. À partir do spin nuclear pode-se obter diversas informações a respeito das estruturas químicas, sendo muito utilizado para a caracterização de novos compostos e a formação de polimorfismo. A amostra a ser analisada é inserida em tubos de vidro longos e delgados que são colocados em câmara entre polos do magneto que passará em uma rápida rotação através de uma turbina, essa transição permite uma variação do campo magnético em um pequeno intervalo, situação que é possível fazer a leitura das variações através do receptor frequência de rádio e transmiti-las para um registrador (LOZANO LS, 2005).

\section{CONSIDERAÇÕES FINAIS}

O domínio sobre as diversas formas de cristais que um fármaco pode adquirir, dependendo das condições físico-químicas de síntese de fármaco, é denominado de polimorfismo. O fenômeno do polimorfismo deve ser considerado como um importante desafio para o processo de síntese, sendo que o controle do processo de desenvolvimento de medicamentos é necessário, de maneira a garantir a farmacocinética e a farmacodinâmica dos medicamentos. Muitos fármacos podem apresentar polimorfos durante 0 desenvolvimento do fármaco, assim como durante a estocagem e por alguma alteração físico-química de produção, por conseguinte, o comprometimento e integração de farmacovigilância são imprescindíveis diante 
dos fármacos polimórficos. Outros importantes aspectos do conhecimento do polimorfismo de fármacos é que novos princípios ativos podem ser patenteados e descobertos, uma vez que pode ser obtido um cristal mais estável, mais seletivo, com melhor biodisponibilidade e que oferecem também menores efeitos colaterais. Muitos fármacos a depender das características físico-químicas de produção submetidos, apresentam polimorfos ainda desconhecidos e não determinados quanto as suas efetividades ou toxicidades. Diante do exposto no artigo de revisão são notórias as influências e o riscos que o polimorfismo apresenta para as indústrias farmacêuticas, exigindo um trabalho de produção química, identificação e controle de medicamentos rigorosos. Além do mais, a indústria farmacêutica e a ANVISA têm como prerrogativa estabelecer parcerias junto aos hospitais, drogarias, de modo a controlar também os fármacos polimórficos em nível de farmacovigilância.

\section{REFERÊNCIAS}

1. ARAUJO GLB. Caracterização no estado solido dos polimorfos de Tibolona, Universidade de São Paulo, 2009 p. 39 202.

2. ARAUjo GlB, PitAluga AJ, GutierReZ SM et al. Polimorfismo na produção de medicamentos, Revista de Ciências Farmacêutica Básica e Aplicada, São Paulo, 2012; 33(1): 27-36.

3. BAUER J, SPANTON S, HENRY R et al. Ritonavir: An Extraordinary Example of Conformational Polymorphism. Pharmaceutical Research, 2001; 18(6): 1-8.

4. BOERRIGTER SXM, VAN DEN HOOGENHOF CJM, MEEKES, $\mathrm{H}$ et al. In Situ Observation of epitaxial polymorphic nucleation of the Model Steroid Methyl Analogue. Norethindrone. Journal of Physical Chemistry, 2002; 106(18): 47254731.

5. BORTOLLUZZI ALML. Estudo do polimorfismo em diferentes fármacos de interesse para a indústria farmacêutica: cimetidina, mebendazol e paracetamol, Universidade Federal de Santa Catarina, Florianópolis, 2011; 25-103.

6. CAABEIRO RF, FORNELIO CA, GONZALES JA et al. Experimental chemotherapy and toxicity in mice of three mebendazole polymorphic forms. Chemotherapy, 1987; 33:266-71.

7. CAPUCHO HC, MASTROIANNI PC, CUFFINI S. Farmacovigilância no Brasil: a relação entre polimorfismo de fármacos, efetividade e segurança dos medicamentos. Revista Cientifica Farmacêutica Básica, São Paulo, 2008; 277-283.

8. CASTRO RAE, MARIA TMR, ÉVORA AOL et al. Estudo de Dois Polimorfos da Pirazinamida Por Calorimetria Diferencial de Varrimento de Espectroscopia de Infravermelho. Química e Ensino, 2013; 53-58.

9. COSTA J, FRENO M, GUZMÁN L et al. Formas polimórficas del mebendazol: aspectos analíticos y toxicidad. Cir. Farm, 1991;49: 415-26.

10. DUTRA KCS. Preparação e Caracterização de Estruturas Polimórficas da Tolbutamida e Nifedipina, Universidade Federal Fluminense, 2005, Niterói, p.26.

11. FROEHLICH PE, GASPAROTTO FS. Mebendazol: identificação das formas polimórficas em diferentes matériasprimas e medicamentos (referência e genéricos) disponíveis no mercado nacional. Revista de Ciências Farmacêuticas Básica e Aplicada, 2005; 26(3): 205-210.

12. GORKOVENKO EA, KICHANOV SE, KOZLENKO DP et al. The Pressure- Induced Polymorphic Transformations In Fluconazole, Journal Of Pharmaceutical Sciences, Russia, 2015; 4164-4169.

13. GUTIERREZ AS. Aplicação da difração de raio $X$ por policristais e do método de Rietveld de refinamento de estruturas cristalinas no estudo de polimorfismo cristalinos de fármacos, Universidade Estadual Paulista Campus de Araraquara, Araraquara, 2010; 24-161.

14. HIENDRAWAN S, WIDJOJOKUSUMO E, VERIANSYAH B et al. Pharmaceutical Salts of Caverdilol: Polymorphism and physicochemical Properties, American Association of Pharmaceutical Scientises, 2016.

15. LACHMAN L, LIEBERMAN HA, KANIG JL. Teoria e pratica na indústria farmacêutica, Lisboa: Fundação Calouste Gulbenkian, 2001; 1: 305.

16. LOZANO LS. Avaliação do Polimorfismo de Fármacos Utilizados para Produção de Medicamentos Genéricos no Brasil, Universidade Federal de Rio Grande do Sul, Porto Alegre, 2005; 1-93.

17. MARTINS FT. Aplicações Tecnológicas do Polimorfismo Farmacêutico, Universidade Federal de Goiás, Goiânia, 2010: 9-21.

18. MEIRELLES LMA. Estabilidade de Medicamentos: Estado da Arte, Revista Eletrônica de Farmácia, Piauí, 2014: 1214.

19. PISEGNA GL, GILSON DFR, BUTLER IS. High- pressure infrared and Raman studies of polymorphism in pharmaceutical compounds: Spironalactone, Forms I and II. Journal of Molecular Structure, Canada, 2014: 146-150.

20. SLÁDKOVÁ V, DAMMER O, KRATOCHVÍL B. Solid Forms of Tenofovir Disoproxil Fumarate, Their Mutual Conversion, and Stabilization of Form I in Formulation. Journal of Pharmaceutical Sciences, 2016: 3136-3142. 